\title{
Methylphenidate improves response inhibition but not reflection-impulsivity in children with attention deficit hyperactivity disorder (ADHD)
}

\author{
Elise E. DeVito • Andrew D. Blackwell • Luke Clark • \\ Lindsey Kent • Anna Maria Dezsery • \\ Danielle C. Turner • Michael R. F. Aitken • \\ Barbara J. Sahakian
}

Received: 31 July 2008 /Accepted: 9 September 2008 / Published online: 26 September 2008

(C) The Author(s) 2008. This article is published with open access at Springerlink.com

\begin{abstract}
Rationale Impulsivity is a cardinal feature of attention deficit hyperactivity disorder (ADHD), which is thought to underlie many of the cognitive and behavioural symptoms associated with the disorder. Impairments on some measures of impulsivity have been shown to be responsive to pharmacotherapy. However, impulsivity is a multi-factorial construct and the degree to which different forms of impulsivity contribute to impairments in ADHD or respond to pharmacological treatments remains unclear.

Objectives The aims of the study were to assess the effects of methylphenidate (MPH) on the performance of children with ADHD on measures of reflection-impulsivity and
\end{abstract}

E. E. DeVito $(\bowtie) \cdot$ A. D. Blackwell • L. Kent • D. C. Turner •

B. J. Sahakian

Department of Psychiatry, University of Cambridge,

P.O. Box 189, Addenbrooke's Hospital,

Cambridge CB2 2QQ, UK

e-mail: eed24@medschl.cam.ac.uk

L. Clark · M. R. F. Aitken

Department of Experimental Psychology,

University of Cambridge,

Cambridge, UK

E. E. DeVito - L. Clark - D. C. Turner • M. R. F. Aitken •

B. J. Sahakian

MRC/Wellcome Trust Behavioural and Clinical Neuroscience Institute, University of Cambridge,

Cambridge, UK

L. Kent $\cdot$ A. M. Dezsery

Section of Child and Adolescent Psychiatry,

University of Cambridge,

Cambridge, UK response inhibition and to compare with the performance of healthy volunteers.

Methods Twenty-one boys (aged 7-13 years) diagnosed with ADHD underwent a double-blind, placebo-controlled trial of MPH $(0.5 \mathrm{mg} / \mathrm{kg})$ during which they performed the Information Sampling Task (IST) and the Stop Signal Task. A healthy age- and education-matched control group was tested on the same measures without medication.

Results Children with ADHD were impaired on measures of response inhibition, but did not demonstrate reflectionimpulsivity on the IST. However, despite sampling a similar amount of information as their peers, the ADHD group made more poor decisions. MPH improved performance on measures of response inhibition and variability of response, but did not affect measures of reflection-impulsivity or quality of decision-making.

Conclusions MPH differentially affected two forms of impulsivity in children with ADHD and failed to ameliorate their poor decision-making on the information sampling test.

Keywords Impulsivity · Reflection-impulsivity . Response inhibition · Methylphenidate - ADHD ·

Stop signal · Decision-making $\cdot$ Cognitive enhancement . Disinhibition · Cognition

\section{Introduction}

Attention deficit hyperactivity disorder (ADHD) is a prevalent neuropsychiatric disorder for which impulsivity (e.g. tendency to interrupt, difficulty awaiting turn) is considered to be a cardinal feature (Barkley 1997; Halperin et al. 1995; Nigg 2000). Children with ADHD are often 
rated by parents, teachers, clinicians and themselves as displaying more 'impulsive' behaviours than their peers (Chamberlain and Sahakian 2006). These clinical symptoms can be quantified using laboratory tasks of impulsivity in children. Given the multi-factorial nature of the impulsivity construct (Evenden 1999; Reynolds 2006), existing tasks fall within three core domains. Inhibition of pre-potent responses can be assessed with the Stop Signal Task (SST; Logan et al. 1984) or with go/no go (Aron and Poldrack 2005) or continuous performance tasks (CPT; Rosvold and Delgado 1956). Delay impulsivity, defined as the choice of a smaller immediate reward over larger delayed reward can be assessed with tasks of temporal discounting or delayof-gratification (Scheres et al. 2006; Schweitzer and Sulzer-Azaroff 1995; Sonuga-Barke et al. 1992). A third domain, which has received less attention, refers to impulsivity at the pre-decisional stage, when the individual gathers and evaluates information. Kagan (1966) first defined the concept of reflection-impulsivity using his Matching Familiar Figures Test (MFFT) where the aim is to determine which of six figures is identical to a standard. Kagan defined 'reflectives' as those subjects with long decision latencies but low error rates, whilst 'impulsives' decided quickly and made more errors. 'Reflectives' not only gather more information, but also use a more systematic approach to comparing the figures than 'impulsives'(Drake 1970). Hyperactive children (i.e. the diagnosis that later became ADHD in the DSM-III) were classed as 'impulsives' more often than their peers on the MFFT (Messer 1976; Sandoval 1977). However, Block et al. (1974) criticised the concept of reflection-impulsivity as defined by the MFFT for disregarding those subjects who perform quickly and well or slowly and poorly simply because they do not meet the original definition of 'reflective' or 'impulsive' (Block et al. 1974).

An alternative measure of reflection-impulsivity, the Information Sampling Task (IST; (Clark et al. 2006)), has recently been developed to circumvent some of the criticisms of the MFFT and to allow for a more direct analysis of information sampling and quality of decisionmaking. In this task, subjects sample information from a covered array of 25 boxes in order to decide which of two colours lies in the majority under the board. Although the subjects are able to sample information until a point of complete certainty, healthy young adults typically make their decision at a point of uncertainty, such that there is around a $20 \%$ chance of error. Substance users dependent upon stimulants or opiates, as well as regular cannabis users, show impulsivity and lack of sufficient sampling (Clark et al. 2006, 2008). One objective of the present study was to examine task performance on the IST in children with ADHD in a case-control design. A further aim was to characterise the effects of ADHD treatment on this variety of impulsivity.
Methylphenidate (MPH) is the first-line treatment for ADHD and acts to increase dopamine and noradrenaline availability by blocking their reuptake (Berridge et al. 2006). MPH remediates impulsivity in children and adults with ADHD on behavioural symptom ratings (Solanto et al. 2001) as well as laboratory tests of response inhibition (Aron et al. 2003; Konrad et al. 2004; Tannock et al. 1989) and the MFFT (Brown and Sleator 1979).

In this study, we compared the effects of MPH on two forms of impulsivity: response inhibition measured by the SST (Aron et al. 2003) and reflection-impulsivity measured by the IST (Clark et al. 2006).

\section{Methods}

Methods and materials

Parental written informed consent and ethics committee approval were obtained. Patients were regular attendees at a childhood ADHD outpatient clinic and were consecutively referred to the study by their psychiatrists. All patient participants had undergone a psychiatrist-led 3-h clinical assessment based on Kiddie-Schedule for Affective Disorders and Schizophrenia and provided developmental and family histories and teacher reports as part of standard care at the outpatient clinic. All met the DSM-IV guidelines for ADHD combined type, including pervasiveness of symptoms (APA 1994) and were stabilised on MPH for therapeutic purposes prior to their referral to the study. ADHD patients $(N=21)$ were males, aged 7-13 years with no primary learning disabilities or concomitant neurological, psychiatric or behavioural disorders (except history of oppositional defiant disorder; $N=14$ ). A healthy control group (HC; $N=21$; aged 7-12 years) was recruited with posters from the local community. Groups were matched for age and years of education.

The ADHD boys underwent a double-blind, placebocontrolled, cross-over design of placebo or a single $0.5-\mathrm{mg} /$ $\mathrm{kg}$ dose (rounded up to the nearest $0.5 \mathrm{mg}$ ) of immediaterelease MPH delivered in capsules. One child received $0.25 \mathrm{mg} / \mathrm{kg}(10 \mathrm{mg})$ since his regular MPH dose with therapeutic efficacy was low in comparison with his higher bodyweight. Participants abstained from MPH for 21-28 h (approximately five to seven half-lives) prior to testing sessions. MPH reaches peak plasma concentration in approximately $2 \mathrm{~h}$ (Volkow et al. 2002). Questionnaires were completed once at the start of a visit to avoid treatment effects. Neuropsychological testing began at least $11 / 2 \mathrm{~h}$ after capsules were ingested. HCs attended two sessions but received no capsules. Sessions were separated by approximately 1-2 weeks. For case-controlled comparisons, each HC participant had his first or second visit randomly designated as 
a 'placebo' control session. HC data was thus matched for age, visit and test order to the ADHD-placebo sessions.

Participants completed the Junior $\mathrm{I}_{6}$ questionnaire (Eysenck 1981), which is designed to measure self-reported impulsive, venturesome and empathetic traits, as well as the Mood and Feeling Questionnaires (Wood et al. 1995), a measure of depressive symptoms. Parents completed disruptive behaviour questionnaires [Achenbach Child Behaviour Checklist (Achenbach 1991); Conners Symptom Behaviour Checklist (Conners 1989)] based on their sons' behaviour without medication. The order of task administration and task conditions were counter-balanced and matched between groups.

\section{Information Sampling Task}

The IST (Clark et al. 2006) is a measure of 'reflectionimpulsivity'. Subjects were told they would be presented with ten trials in each of two conditions [fixed win (FW), decreasing win (DW)] and that the task would last for $10 \mathrm{~min}$. On each trial, a $5 \times 5$ matrix of grey boxes with two larger coloured panels below it was presented. Subjects were told to press on a box to reveal its colour and to open as many boxes as they chose prior to deciding which of the two colours they thought was in the majority amongst the 25 boxes. Boxes remain open for the duration of the trial to minimise working memory load. To register their decision, they pressed on one of the two coloured panels displayed. In the FW condition, if the subject chose correctly, they earned 100 points, but lost 100 points if they chose incorrectly. In the DW condition, subjects begin with the ability to win 250 points, but with every box they opened the amount they could win decreased by 10 points. An incorrect choice was still penalised with a loss of 100 points regardless of the amount of boxes opened. Therefore, in the FW condition, extensive information sampling prior to decision was optimal, whilst in order to gain points in the DW condition the subject must adjust to sample less information. A variable inter-trial interval was used such that the minimum trial duration was $30 \mathrm{~s}$ (e.g. if the subject responded after $10 \mathrm{~s}$, the inter-trial interval would be $20 \mathrm{~s}$ ). This feature was included to prevent unduly rapid responses leading to shorter task duration and thus an earlier escape of the experimental setting. This task is described in more detail by Clark et al. (2006).

Within each condition (FW and DW), there are five key measures. 'Boxes opened' is the number of boxes they chose to open, which represents the amount of information they sample prior to making a decision. 'Box adjustment' is the differences in number of boxes opened in the FW versus DW conditions and represents the degree to which subjects adjust their behaviour to the reward contingencies. 'Quality of information' is the probability that the colour in the visible majority at the time of choice is the true majority colour given the number of boxes remaining covered and assuming each uncovered box has a 0.5 probability of being each colour. For details of the calculation, see Clark et al. (2006). 'Quality of information' is the primary measure for information sampling on this task since it accounts for the amount of boxes opened as well as the proportion of each colour visible in the uncovered areas of the matrix at the time of decision. 'Choice latency' is the time between opening the final box and guessing which colour is in the majority within the matrix. A 'poor decision' is when the colour in the minority amongst the unveiled boxes is chosen.

A further measure, 'sampling errors', was employed to confirm that the model of 'reflection-impulsivity' provides a reasonable account of the data. This measure was not used to analyse subjects' task performance. Sampling errors occur when the colour in the visible majority is chosen, but by chance is not the colour within the majority of the matrix. In a reflection-impulsivity task, the long-term probability of sampling errors should be inversely related to the quality of information: such errors are more likely to occur when less information is sampled (i.e. fewer boxes are opened).

\section{Stop Signal Task}

The SST (Aron et al. 2003) is a well-established measure of response inhibition. On each trial, subjects are asked to make a rightward or leftward button press as quickly as possible when presented with an arrow in the centre of the screen pointing in the corresponding direction (go stimulus). On a minority of the trials (25\%), an auditory 'stop signal' beep is presented at a variable delay (stop signal delay, SSD) following the go stimulus. Subjects are instructed to inhibit their responses on the trials with a stop signal beep. Subjects complete five blocks of 64 trials; each of the SSD is titrated to the subject's ability using a tracking algorithm (Osman et al. 1990) to converge on 50\% successful stop rate. The primary outcome measure is stop signal reaction time (SSRT), an estimate of the subject's capacity for inhibiting pre-potent motor responses. To ensure accurate estimation of the SSRT for subjects who did not converge exactly on $50 \%$ successful stops, the SSRT was computed using the integration method (Logan 1994). The SSRT as estimated by the integration method produced qualitatively similar results as the race-model estimation in this data set. Additional measures of interest are the median (median go) and standard deviation (SD; variability go) of the go reaction times.

Statistical analyses

$T$ tests or non-parametric equivalents (Wilcoxon signed ranks or Mann-Whitney $U$ tests), where appropriate, and 
chi-squared analysis compared ADHD-placebo with ADHDMPH and healthy controls on IST and SST measures, rating scales and demographic variables. Data were examined for statistical outliers (defined as $>3 \times$ interquartile range outside either quartile boundary) before analysis. The exclusion of outliers did not alter the pattern or significance of results. On measures that significantly improved on MPH, ADHD-MPH was compared directly with the HC group. Effect sizes were calculated as $d=\left(\mu_{1}-\mu_{2}\right) / \sqrt{ }\left[\left(\sigma_{1}{ }^{2}+\sigma_{2}{ }^{2}\right) / 2\right]$ (Dunlap et al. 1996). Exploratory correlations (Pearson's or Spearman's) were carried out between the core task measures that showed significant impairments or changes on MPH and behavioural ratings. Due to the large number of correlations, only those significant to $p<0.01$ will be reported.

\section{Results}

ADHD and HC groups were matched for age $(t=-0.872$, $p=0.388 ; \operatorname{mean}_{\mathrm{ADHD}}=10.00, \mathrm{SD}_{\mathrm{ADHD}}=2.04 ; \operatorname{mean}_{\mathrm{HC}}=$ $\left.10.47, \mathrm{SD}_{\mathrm{HC}}=1.43\right)$, test order $(t=-0.302, p=0.765)$, visit used $(t=0.302, p=0.765)$, days between visits $(t=-0.710$, $p=0.482)$, years of education $(t=-1.063, p=0.297)$ and distribution of younger (7-10) versus older (11-13) children $\left(d f=1, \chi^{2}=1.52, p=0.22\right)$. The ADHD group had significantly higher disruptive behaviour ratings (Achenbach $_{\text {Total }} t=6.795, p<0.001$; Conners Total $t=8.777, p<$ $0.001)$ and a trend towards higher depressive symptoms (Mood and Feeling Questionnaire $t=1.852 ; p=0.072$ ). Two ADHD subjects who received MPH on session 1 did not return for their second sessions and were excluded from the analyses.
Information Sampling Task

Participants demonstrated sensitivity to the task contingencies by opening fewer boxes in the DW condition than the FW condition (HC $z=-3.980, p<0.001$; ADHDplacebo $z=-3.680, p<0.001$; ADHD-MPH $z=-3.920, p<$ $0.001)$. A core criterion for a test of reflection-impulsivity is that the extent of information sampling should predict the accuracy of the eventual decision. This criterion was generally evident in the data as negative correlations between sampling errors and the number of boxes opened in the FW condition (HC $r=-0.771, p<0.001 ;$ ADHD-placebo $r=-0.634, p=$ 0.005; ADHD-MPH $r=0.109, p=0.648$ ).

IST group effects (ADHD-placebo versus healthy controls)

ADHD-placebo did not differ significantly from healthy controls in either condition on the amount of boxes opened (FW $z=-0.620, p=0.549, d=0.18$; DW $z=-0.930, p=$ $0.364, d=0.18)$, box adjustment $(z=-0.592, p=0.568, d=$ 0.28 ), choice latency ( $\mathrm{FW} z=-1.155, p=0.257, d=0.37$; DW $z=-1.465, p=0.148, d=0.51$ ) or quality of information available at the time of choice (FW $z=-0.509, p=0.626, d=$ 0.13; DW $z=1.296, p=0.202, d=0.40$; see Table 1). In both conditions, ADHD-placebo made significantly more poor decisions (i.e. chose the colour in the visible minority) than the HC group (FW $z=-2.954, p=0.006, d=1.06$; DW $z=-3.097$, $p=0.003, d=0.90$; see Fig. 1a).

\section{IST drug effects (ADHD-MPH versus ADHD-placebo)}

MPH did not significantly alter the performance of the ADHD group on the key IST measures. ADHD-MPH did not

Table 1 IST key measures

\begin{tabular}{llrr}
\hline IST measure & Group/session & Fixed win, mean (SEM) & Decreasing win, mean (SEM) \\
\hline Boxes opened & HC & $16.83(1.00)$ & $7.66(0.65)$ \\
& ADHD-placebo & $17.81(1.45)$ & $6.92(1.13)$ \\
Quality of information & ADHD-MPH & $16.26(1.53)$ & $5.83(1.03)$ \\
& HC & $0.86(0.02)$ & $0.70(0.01)$ \\
& ADHD-placebo & $0.87(0.03)$ & $0.67(0.02)$ \\
Choice latency $^{a}$ & ADHD-MPH & $0.83(0.12)$ & $0.66(0.09)$ \\
& HC & $3.34(0.80)$ & $1.78(0.15)$ \\
Poor decisions & ADHD-placebo & $4.72(0.92)$ & $2.23(0.24)$ \\
& ADHD-MPH & $3.96(0.91)$ & $2.38(0.38)$ \\
& HC & $0.38(0.15)$ & $0.62(0.03)$ \\
& ADHD-placebo & $1.33(0.26)$ & $1.94(0.37)$ \\
\end{tabular}

$H C$ healthy control group, $A D H D$-placebo attention deficit hyperactivity group on placebo, $A D H D-M P H$ attention deficit hyperactivity group on methylphenidate

${ }^{a}$ Latency measures are reported in seconds 
Fig. 1 Group and drug effects on IST poor decisions and SST SSRT

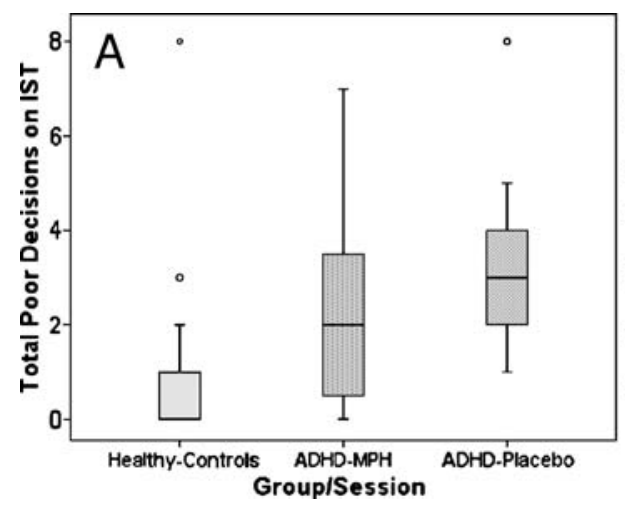

significantly differ from ADHD-placebo visits on the number of boxes opened (FW $z=-0.828, p=0.407, d=0.25$; DW $z=-1.045, p=0.296, d=0.24)$, box adjustment $(t=-0.251$, $p=0.805, d=0.07$ ), choice latency ( $\mathrm{FW} z=-1.328, p=0.184$, $d=0.20$; DW $z=-0.240, p=0.811, d=0.11$ ), quality of information available at the time of choice (FW $z=-1.086$, $p=0.278, d=0.35$; DW $z=-0.806, p=0.420, d=0.12$ ) or number of poor decisions ( $\mathrm{FW} z=-1.078, p=0.281, d=0.32$; DW $z=-0.405, p=0.685, d=0.12$ ).

IST effects of trial type (poor decision trials versus sensible decision trials)

To further investigate the higher rates of poor decisions in the ADHD group on placebo, post hoc analyses were carried out to compare trials where poor decisions were made with sensible decision trials within each group and condition (see Table 2).

Within ADHD-placebo, poor-quality decision trials did not differ from sensible trials in terms of choice latency (FW $z=-0.245, p=0.807, d=0.10$; DW $z=-0.785$, $p=0.433, d=0.10)$. However, the quality of information available at the time of choice was lower for ADHDplacebo on poor decision trials than sensible trials in the FW condition only (FW $z=-2.29, p=0.022, d=0.66$; DW $z=-0.722, p=0.470, d=0.36$ ). Within ADHD-MPH, poor and sensible decision trials did not differ significantly in terms of quality of information available at the time of choice (FW $z=0.000, p=1.000, d=0.30$; DW $z=-0.80, p=$ $0.424, d=0.35$ ), but ADHD-MPH had significantly shorter choice latencies on poor-quality decision trials than sensible trials in the FW condition only (FW $z=-2.045$, $p=0.041, d=0.67$; DW $z=-1.511, p=0.131, d=0.51$ ).

In addition, ADHD-MPH did not significantly differ from ADHD-placebo on choice latency or quality of information measures within poor or sensible decision trials in either condition.

IST effects of task duration on probability of correct choice

A post hoc analysis was performed to determine whether higher rates of errors in the ADHD group were related to fatigue or loss of motivation across the duration of the task. The probability that the subjects' choices were correct given the information available at the time of decision was calculated for each trial using a variant of the equation for 'quality of information'. Whilst 'quality of information' represents the probability of being correct if the subject chooses the colour in the visible majority at the time of decision (i.e. the 'sensible' choice), the calculation for probability of correct choice instead considers the colour actually chosen by the subject. Therefore, poor decisions always have a probability of being correct of less than 0.5 .

Table 2 IST poor decision trials versus sensible decision trials

\begin{tabular}{|c|c|c|c|c|}
\hline IST measure & Group/session & Trial decision & Fixed win, mean (SEM) & Decreasing win, mean (SEM) \\
\hline \multirow[t]{4}{*}{ Quality of information } & \multirow[t]{2}{*}{ ADHD-placebo } & Poor & $0.79(0.05)$ & $0.64(0.03)$ \\
\hline & & Sensible & $0.89(0.02)$ & $0.67(0.02)$ \\
\hline & \multirow[t]{2}{*}{ ADHD-MPH } & Poor & $0.90(0.05)$ & $0.64(0.04)$ \\
\hline & & Sensible & $0.85(0.02)$ & $0.68(0.02)$ \\
\hline \multirow[t]{4}{*}{ Choice latency $^{\mathrm{a}}$} & \multirow[t]{2}{*}{ ADHD-placebo } & Poor & $5.04(1.67)$ & $2.37(0.35)$ \\
\hline & & Sensible & $4.53(0.91)$ & $2.25(0.27)$ \\
\hline & \multirow[t]{2}{*}{ ADHD-MPH } & Poor & $2.32(0.54)$ & $2.00(0.31)$ \\
\hline & & Sensible & $4.50(0.90)$ & $2.93(0.53)$ \\
\hline
\end{tabular}

ADHD-placebo attention deficit hyperactivity group on placebo, $A D H D-M P H$ attention deficit hyperactivity group on methylphenidate

${ }^{a}$ Latency measures are reported in seconds 
This novel measure was introduced because it accounts for poor decisions but the data meets the assumptions of normality necessary for inclusion in an analysis of variance (ANOVA). A mixed-model ANOVA measured the change in the probability of being correct across the within-subject factors of trial (1-10) and condition (FW, DW) and between-subject factors of group (ADHD-placebo, HC) and condition order (FW-DW, DW-FW). There were significant main effects of group $(F=5.804, p=0.021)$ and trials $(F=7.668, p<0.001)$ on the probability of being correct. The main effect of trials was due to a fluctuation from trial to trial (the precise sequence of colours revealed in a session was taken from the same pseudo-random sequence for all participants), rather than a clear trend across the task duration. There were no significant trial by group $(F=1.434, p=0.186)$, trial by condition order $(F=1.231, p=$ $0.283)$ or trial by group by condition order $(F=1.469, p=$ 0.168 ) interactions. Thus, this analysis provides no evidence to suggest that group differences arose through differences in fatigue or motivation over the course of a session.

\section{Stop Signal Task}

SST group effects (ADHD-placebo versus healthy controls)

Compared with the HC group on the SST, ADHD-placebo had significantly slower median go reaction time $(F=3.20, p=$ $0.005, d=1.00)$, larger SD of go reaction times $(z=-4.049, p<$ $0.001, d=1.53)$ and longer SSRTs $(t=3.78, p=0.001, d=1.28$; see Table 3).

\section{SST drug effects (ADHD-MPH versus ADHD-placebo)}

Compared with ADHD-placebo, ADHD-MPH had less variable go reaction times $(z=-3.501, p<0.001, d=1.10)$ and shorter SSRTs $(t=-4.169, p=0.001, d=1.54)$, but did not

Table 3 SST key measures

\begin{tabular}{lll}
\hline SST measure & Group/session & Mean (SEM) \\
\hline SSRT & HC & $263.93(20.0)$ \\
& ADHD-placebo & $376.84(22.01)$ \\
Median go RT & ADHD-MPH & $242.80(19.30)$ \\
& HC & $442.76(22.76)$ \\
& ADHD-placebo & $548.42(30.86)$ \\
SD go RT & ADHD-MPH & $520.21(26.42)$ \\
& HC & $175.46(73.61)$ \\
& ADHD-placebo & $530.18(77.39)$ \\
& ADHD-MPH & $225.36(28.10)$ \\
\hline
\end{tabular}

$H C$ healthy control group, $A D H D$-placebo attention deficit hyperactivity group on placebo, $A D H D-M P H$ attention deficit hyperactivity group on methylphenidate, SSRT stop signal reaction time

${ }^{\text {a }}$ Reaction time (RT) measures are reported in milliseconds significantly differ on median go reaction times $(t=-1.573$, $p=0.133, d=0.23$.

SST medicated ADHD group effects (ADHD-MPH versus healthy controls)

ADHD-MPH and HC groups were only directly compared on measures that were significantly affected by MPH. ADHD on medication (ADHD-MPH) no longer significantly differed from healthy controls on SSRT $(t=-4.75$, $p=0.638, d=0.26$; see Fig. $1 \mathrm{~b}$ ) or $\mathrm{SD}$ of go reaction time $(z=-1.648, p=0.099, d=0.50)$.

\section{Associations between measures of impulsivity}

Measures from the SST (SSRT, SD of go reaction time) and IST (quality of information, poor decisions) were compared with each other as well as with self-rated trait ( $\mathrm{I}_{6}$ impulsivity, venturesomeness, empathy subscales) and parent-rated behavioural (Conners total score, Achenbach total score) questionnaires.

In the ADHD group, higher self-rated impulsivity $\left(\mathrm{I}_{6}\right)$ was associated with reduced quality of information tolerated in the FW condition on placebo $\left(r_{18}=-0.661\right.$, $p=0.003$; see Fig. 2). Higher self-rated trait venturesomeness $\left(\mathrm{I}_{6}\right)$, which involves questions in regard to risky but hedonic adventures, was associated with better response inhibition (SSRT) in the ADHD group on MPH (ADHD-

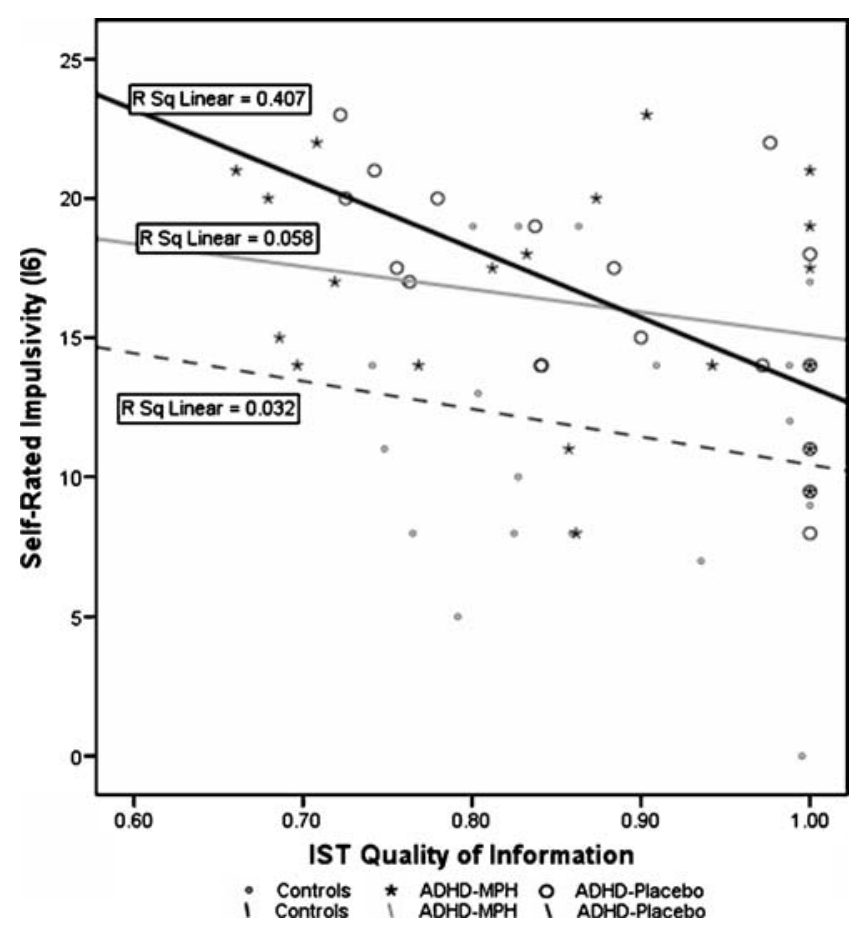

Fig. 2 More uncertainty tolerated prior to decision-making associated with self-rated impulsivity in ADHD-placebo 


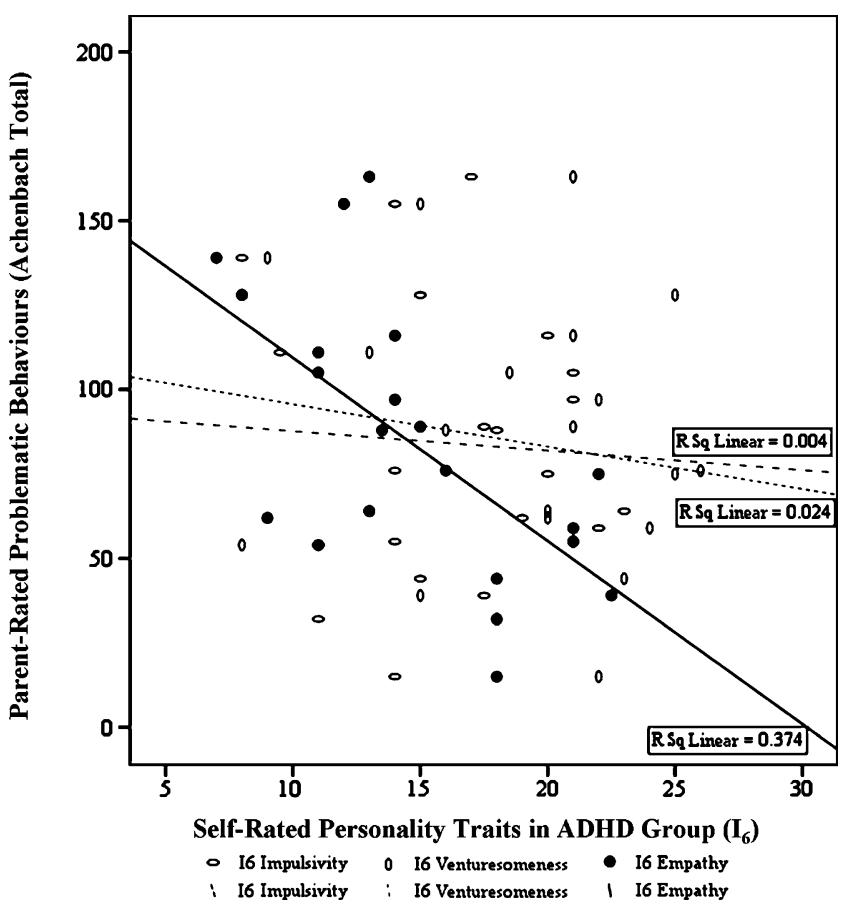

Fig. 3 Self-rated empathy not impulsivity associated with behaviour ratings

MPH; $\left.r_{19}=-0.605, p=0.006\right)$. The reason for this association requires further investigation.

In the ADHD group, self-rated empathy, but not impulsivity or venturesomeness $\left(\mathrm{I}_{6}\right)$, correlated negatively with parent-rated disruptive behaviours (Achenbach ${ }_{\text {Total }}$ $r=-0.612, p=0.003)$ and ADHD symptoms (Conners ${ }_{\text {Total }}$ $r=-0.599, p=0.005$; see Fig. 3).

\section{Discussion}

On the SST, response inhibition was improved by MPH as was variability of reaction time. These findings replicate previous results in children (Konrad et al. 2004; Lijffijt et al. 2006; Tannock et al. 1989) and adults (Aron et al. 2003) with ADHD. On the IST, ADHD children did not differ from healthy control children in terms of the amount of information they sampled, the degree of uncertainty tolerated prior to making a decision or the speed at which they made their final choice. However, the ADHD group on placebo made significantly more poor decisions than the healthy controls. MPH did not significant affect any of the primary IST measures. Post hoc analyses revealed that within the ADHD group on placebo, these subjects tolerated a lower probability of being correct on the trials where they made poor decisions, signifying a degree of cognitive impulsivity contributing to their higher error rate. These problems were not ameliorated by MPH. The ADHD group on MPH took less time to make their final decision on the trials where they made poor decisions compared with the trials where they chose the colour in the visible majority, perhaps suggesting less attention to the trials where errors were made.

The lack of correlations between IST and SST measures is consistent with the idea that response inhibition and reflection-impulsivity are separate forms of impulsivity (Evenden 1999). Despite the widely accepted value of both rating scales and laboratory measures, it is common to find a lack of statistical associations between these measures of impulsivity (Lijffijt et al. 2004; Swann et al. 2002). This has been attributed to unreliable or biased reporting on ratings scales (e.g. children's poor insight into their personality or behaviour; tendency for adults to respond with socially appropriate answers) or to the different forms of measurement tapping into different aspects of impulsivity (e.g. trait versus state). Therefore, it is significant that in the children with ADHD off medication, toleration of less quality of information prior to deciding was correlated strongly with their self-rated impulsivity. Parent ratings of disrupted behaviours did not relate to IST or SST measures, they were also not related to children's self-rated impulsivity. The strong association between children's self-rated empathy and parent-rated behavioural scales suggests that poor social perception or difficulty appreciating the impact of one's behaviour on others (Clark et al. 1999) may contribute to disruptive behaviours in ADHD.

This study is the first demonstration of the IST in healthy children or children with ADHD, both on and off MPH. The lack of group effects on some measures of IST was interesting, given that impulsive responding (e.g. less information sampling prior to deciding) was previously demonstrated in current and former drug users (Clark et al. 2006, 2008) as well as adults with major depressive disorder (Taylor Tavares et al. 2007) on this task. The children appeared to comprehend the task requirements by adjusting their information sampling according to the condition (FW, DW) and choosing the colour in the visible majority on the majority of trials. Poor quality of decisionmaking distinguished children with ADHD from their peers, but was not accompanied by significantly less information sampling or differences in speed of decisionmaking. Therefore, the impairment in quality of decisionmaking on IST does not appear to be attributable to greater motor impulsivity in this context. The fact that patients do tolerate a lower quality of information on the trials in which they make errors may suggest that fluctuations in their attention or cognitive impulsivity across the duration of the task may contribute to their poor-quality decisions. These effects cannot easily be explained by reduced motivation as a result of fatigue in the ADHD group since statistical analyses showed no group differences on the performance effects of time on task. In regards to the limitations of this 
study, these findings await replication and extension to a larger group of ADHD patients including other ADHD subgroups and females.

The traditional concept of reflection-impulsivity predicts that impulsive subjects would respond quickly (i.e. prior to gathering sufficient information) and consequently make more errors than reflective subjects (Kagan 1966). However, we demonstrated increased errors without significantly lower levels of information sampling in the ADHD group. These findings are consistent with Block et al. (1974) who found that symptoms associated with hyperactivity in healthy children were most commonly observed in those subjects who made many errors on the MFFT despite responding slowly. In fact, subjects who responded quickly and inaccurately were not more likely to display hyperactive traits (Block et al. 1974). Although the prevalent method of analysing the MFFT incorporates latency and error rates, latency can be influenced by minor manipulations, such as changing task instructions to encourage slower responding. In contrast, error rates could only be improved by teaching systematic strategies or presenting the figures as component parts to encourage systematising (Block et al. 1974). Therefore, they concluded that error rates are a more reliable and stable measure of cognitive style than latency.

The lack of impulsive information sampling in children with ADHD with accompanying high error rates suggests they may be failing to use the available information to their advantage as well as their peers. This tendency is not ameliorated by MPH, despite its robust effects on other forms of impulsivity and response control measured in the same session. Poor quality of decision-making resistant to treatment with MPH has been previously demonstrated in an earlier study of children with ADHD on the Cambridge Gamble Task (DeVito et al. 2008). Methods for improving decisionmaking are, therefore, an important target for pharmacological as well as non-pharmacological treatments.

\section{Conclusions}

Although MPH reversed the impairments in motor control and response inhibition in children with ADHD, it did not significantly improve their poor quality of decision-making.

Acknowledgements The authors thank the participants and their families for generously volunteering their time. We wish to acknowledge the valuable contributions of Dr. Karen Ersche and Dr. Claire H Salmond for the input into the set-up and design of the study as well as Dr. Sharon Morein-Zamir for statistical advice regarding the SST. We thank the administrative staff at both testing facilities for their assistance. This work has been supported by a
Wellcome Trust Programme Grant (\#076274/Z/04/Z) to TW Robbins, BJ Everitt, AC Roberts and BJ Sahakian and was completed within the University of Cambridge Section of Child and Adolescent Psychiatry testing facilities and the University of Cambridge Behavioural and Clinical Neuroscience Institute (BCNI), which is supported by a joint award from the Medical Research Council (UK) and the Wellcome Trust (G001354). EE DeVito was funded by a Pinsent Darwin Studentship, DC Turner was funded by the Wellcome Trust. BJ Sahakian consults for Cambridge Cognition and a number of pharmaceutical companies. L Clark and DC Turner consult for Cambridge Cognition. Following the completion of the study, AD Blackwell was employed by Cambridge Cognition. EE DeVito, L Kent, AM Dezsery and MRF Aitken report no biomedical financial interests or potential conflicts of interest.

Open Access This article is distributed under the terms of the Creative Commons Attribution Noncommercial License which permits any noncommercial use, distribution, and reproduction in any medium, provided the original author(s) and source are credited.

\section{References}

Achenbach TM (1991) Integrative guide to the $1991 \mathrm{CBCL} / 4-18$, YSR, and TRF profiles. Department of Psychology, University of Vermont, Burlington, VT

APA (1994) Diagnostic and statistical manual of mental disorders, 4th edn. American Psychiatric Association, Washington, DC

Aron AR, Poldrack RA (2005) The cognitive neuroscience of response inhibition: relevance for genetic research in attentiondeficit/hyperactivity disorder. Biol Psychiatry 57:1285-1292

Aron AR, Dowson JH, Sahakian BJ, Robbins TW (2003) Methylphenidate improves response inhibition in adults with attentiondeficit/hyperactivity disorder. Biol Psychiatry 54:1465-1468

Barkley RA (1997) Behavioral inhibition, sustained attention, and executive functions: constructing a unifying theory of ADHD. Psychol Bull 121:65-94

Berridge CW, Devilbiss DM, Andrzejewski ME, Arnsten AF, Kelley AE, Schmeichel B, Hamilton C, Spencer RC (2006) Methylphenidate preferentially increases catecholamine neurotransmission within the prefrontal cortex at low doses that enhance cognitive function. Biol Psychiatry 60:1111-1120

Block J, Block JH, Harrington DM (1974) Some misgivings about the matching familiar figures test as a measure of reflection impulsivity. Dev Psychol 10:611-632

Brown RT, Sleator EK (1979) Methylphenidate in hyperkinetic children: differences in dose effects on impulsive behaviour. Pediatrics 64:408-411

Chamberlain S, Sahakian BJ (2006) Attention deficit hyperactivity disorder has serious and immediate implications. Educ J 94:35-37

Clark T, Feehan C, Tinline C, Vostanis P (1999) Autistic symptoms in children with attention deficit-hyperactivity disorder. Eur Child Adolesc Psychiatry 8:50-55

Clark L, Robbins TW, Ersche KD, Sahakian BJ (2006) Reflection impulsivity in current and former substance users. Biol Psychiatry 60:515-522

Clark L, Roiser JP, Robbins TW, Sahakian BJ (2008) Disrupted 'reflection' impulsivity in cannabis users but not current or former ecstasy users. J Psychopharmacol (in press)

Conners C (1989) Conners rating scales: CPRS-39, CTRS-39. MultiHealth Systems, North Tonawanda, NY

DeVito EE, Blackwell AD, Kent L, Ersche KD, Clark L, Salmond $\mathrm{CH}$, Dezsery AM, Sahakian BJ (2008) The effects of methyl- 
phenidate on decision making in attention-deficit/hyperactivity disorder. Biol Psychiatry 64:636-639

Drake DM (1970) Perceptual correlates of impulsive and reflective behaviour. Dev Psychol 2:202-214

Dunlap WP, Cortina JM, Vaslow JB, Burke MJ (1996) Meta-analysis of experiments with matched groups or repeated measures designs. Psychol Methods 1:170-177

Evenden JL (1999) Varieties of impulsivity. Psychopharmacology (Berl) 146:348-61

Eysenck SBG (1981) Impulsiveness and antisocial behaviour in children. Curr Psychol Res 1:31-37

Halperin JM, Newcorn JH, Matier K, Bedi G, Hall S, Sharma V (1995) Impulsivity and the initiation of fights in children with disruptive behavior disorders. J Child Psychol Psychiatry 36:1199-1211

Kagan J (1966) Reflection-impulsivity: the generality and dynamics of conceptual tempo. J Abnorm Psychology 71:17-24

Konrad K, Gunther T, Hanisch C, Herpertz-Dahlmann B (2004) Differential effects of methylphenidate on attentional functions in children with attention-deficit/hyperactivity disorder. J Am Acad Child Adolesc Psych 43:191-198

Lijffijt M, Bekker EM, Quik EH, Bakker J, Kenemans JL, Verbaten MN (2004) Differences between low and high trait impulsivity are not associated with differences in inhibitory motor control. J Atten Disord 8:25-32

Lijffijt M, Kenemans JL, ter Wal A, Quik EH, Kemner C, Westenberg H, Verbaten MN, van Engeland H (2006) Dose-related effect of methylphenidate on stopping and changing in children with attention-deficit/hyperactivity disorder. Eur Psychiatry 21:544-547

Logan GD (1994) On the ability to inhibit thought and action: a user's guide to the stop signal paradigm. In: Dagenbach $\mathrm{D}$, Carr TH (eds) Inhibitory processes in attention, memory, and language. Academic, San Diego, pp 180-239

Logan GD, Cowan WB, Davis KA (1984) On the ability to inhibit simple and choice reaction time responses: a model and a method. J Exp Psychol Hum Percept Perform 10:276-291

Messer SB (1976) Reflection-impulsivity: a review. Psychol Bull $83: 1026-1052$

Nigg JT (2000) On inhibition/disinhibition in developmental psychopathology: views from cognitive and personality psychology and a working inhibition taxonomy. Psychol Bull 126:220-246
Osman A, Kornblum S, Meyer DE (1990) Does motor programming necessitate response execution? J Exp Psychol Hum Percept Perform 16:183-198

Reynolds B (2006) A review of delay-discounting research with humans: relations to drug use and gambling. Behav Pharmacol 17:651-667

Rosvold HE, Delgado JM (1956) The effect on delayed-alternation test performance of stimulating or destroying electrically structures within the frontal lobes of the monkey's brain. J Comp Physiol Psychol 49:365-372

Sandoval J (1977) The measurement of the hyperkinetic syndrome in children. Rev Educ Res 47:293-318

Scheres A, Dijkstra M, Ainslie E, Balkan J, Reynolds B, Sonuga-Barke E, Castellanos FX (2006) Temporal and probabilistic discounting of rewards in children and adolescents: effects of age and ADHD symptoms. Neuropsychologia 44:2092-2103

Schweitzer JB, Sulzer-Azaroff B (1995) Self-control in boys with attention deficit hyperactivity disorder: effects of added stimulation and time. J Child Psychol Psychiatry 36:671-686

Solanto MV, Arnsten AFT, Castellanos FX (2001) Stimulant drugs and ADHD. Oxford University Press, Oxford

Sonuga-Barke EJ, Taylor E, Sembi S, Smith J (1992) Hyperactivity and delay aversion-I. The effect of delay on choice. J Child Psychol Psychiatry 33:387-398

Swann AC, Bjork JM, Moeller FG, Dougherty DM (2002) Two models of impulsivity: relationship to personality traits and psychopathology. Biol Psychiatry 51:988-994

Tannock R, Schachar RJ, Carr RP, Chajczyk D, Logan GD (1989) Effects of methylphenidate on inhibitory control in hyperactive children. J Abnorm Child Psychol 17:473-491

Taylor Tavares JV, Clark L, Cannon DM, Erickson K, Drevets WC, Sahakian BJ (2007) Distinct profiles of neurocognitive function in unmedicated unipolar depression and bipolar II depression. Biol Psychiatry 62:917-924

Volkow ND, Fowler JS, Wang G, Ding Y, Gatley SJ (2002) Mechanism of action of methylphenidate: insights from PET imaging studies. J Atten Disord 6(Suppl 1):S31-S43

Wood A, Kroll L, Moore A, Harrington R (1995) Properties of the mood and feelings questionnaire in adolescent psychiatric outpatients: a research note. J Child Psychol Psychiatry $36: 327-334$ 